\title{
Assessing the correct inflation of the endotracheal tube cuff: a larger pilot balloon increases the sensitivity of the 'finger-pressure' technique, but it remains poorly reliable in clinical practice
}

\author{
Antonio Pisano ${ }^{1}\left[\right.$ [ $\cdot$ Luigi Verniero $^{1} \cdot$ Nicola Galdieri ${ }^{1} \cdot$ Antonio Corcione $^{2}$
}

Received: 25 February 2018 / Accepted: 20 May 2018 / Published online: 22 May 2018

○) Springer Science+Business Media B.V., part of Springer Nature 2018

\begin{abstract}
The pilot balloon palpation (or 'finger-pressure') method is still widely used to assess the endotracheal tube cuff inflation, despite consistent evidence of its poor sensitivity in recognizing cuff overinflation. It was recently speculated that this may be related to the lower wall tension (due to the smaller radius) of the pilot balloon as compared with the cuff, according to Laplace's law. To verify this hypothesis and, secondarily, to assess whether the use of a 'large' pilot balloon (identical to the cuff) increases the reliability of this technique, 62 anesthetists (41 experienced anesthesiologists and 21 residents) were asked to estimate the pressure of a cuff inflated to $88 \mathrm{mmHg}$ into a simulated trachea by feeling both a usual and a modified 'large' pilot balloon. A similar test was repeated at $40 \mathrm{mmHg}$. After palpation of the usual pilot balloon, only $35 \%$ of participants (49\% of experienced anesthesiologists and $10 \%$ of residents) recognized considerable overinflation $(88 \mathrm{mmHg}$ ), as compared with $87 \%$ of participants (95\% of experienced anesthesiologists and $71 \%$ of residents) after palpation of the 'large' pilot balloon. Moreover, $89 \%$ of participants (85\% of experienced anesthesiologists and $95 \%$ of residents) believed that pressure was higher in the 'large' balloon than in the normal one. However, only $32 \%$ of participants (51\% of experienced anesthesiologists and none of residents) recognized slight overinflation $(40 \mathrm{mmHg})$ after feeling the 'large' balloon. The pilot balloon size affects the sensitivity of the 'finger-pressure' technique, but it remains poorly reliable with a larger pilot balloon.
\end{abstract}

Keywords Tracheal tube cuff $\cdot$ Airway management $\cdot$ Pressure gauge $\cdot$ Tracheal injury $\cdot$ Ventilator-associated pneumonia

\section{Introduction}

Maintaining an inflation pressure of the endotracheal tube cuff within $15-25 \mathrm{mmHg}$ is pivotal in order to prevent potentially serious complications in intubated patients [1, 2]. In fact, while a cuff pressure greater than $15 \mathrm{mmHg}$ allows adequate mechanical ventilation without gas leaks and prevents secretion aspiration, thus reducing the risk of ventilator-associated pneumonia (VAP), a cuff pressure $>25-30 \mathrm{mmHg}$ may compromise tracheal wall capillary blood flow, possibly leading to mucosal ischemia, ulcerations, tracheomalacia, tracheal stenosis, and even

Antonio Pisano

antoniopisanoMD@ libero.it

1 Cardiac Anesthesia and Intensive Care Unit, AORN 'Dei Colli', Monaldi Hospital, via L. Bianchi, 80131 Naples, Italy

2 Department of Critical Care, AORN 'Dei Colli', via L. Bianchi, 80131 Naples, Italy tracheal rupture or tracheo-oesophageal fistulae [2, 3]. Pilot balloon palpation (the so-called 'finger-pressure' method) is still widely used to assess the adequate inflation of the endotracheal tube cuff, despite many investigations clearly showed its inaccuracy [1, 4-9]. In particular, this technique tends to underestimate the actual intracuff pressure, leading to overinflation. In a recent investigation, Michlig [5] asked 53 anaesthesia practitioners to evaluate a cuff inflated to $88 \mathrm{mmHg}$ (i.e. about 3-4 times the recommended value), and found that only $34 \%$ of them recognized overinflation.

It was lately hypothesized that the poor sensitivity of pilot balloon palpation in recognizing cuff overinflation may be due to the smaller radius of curvature of the pilot balloon as compared with the cuff $[10,11]$. It can be speculated that, in order to estimate the pressure within the pilot balloon, clinicians rely on its wall tension, which is directly proportional to the radius according to Laplace's law [11]. For a spherical bubble (whose wall thickness can be considered as negligible), equation for Laplace's law is: 
$T=\frac{p r}{2}$

where $T$ is wall tension, $p$ is the pressure inside the bubble, and $r$ is the bubble radius.

Although the pressure in the pilot balloon is the same as in the cuff (since the "reaction" pressure exerted by the tracheal wall on the cuff is transmitted unaltered to the pilot balloon, according to Pascal's principle [12]), its wall tension is lower, due to the smaller radius of the pilot balloon as compared with the cuff. Therefore, the pilot balloon will always appear more 'flabby' than it is the cuff indeed. Accordingly, it was argued that clinicians would recognize overinflation more easily if they could touch the cuff directly, or with a larger pilot balloon [10,11]. In order to verify the hypothesis that the poor accuracy of the 'finger-pressure' method can be related to the smaller size of the pilot balloon as compared with the cuff and, secondarily, to assess whether the elimination of this difference may be sufficient to increase the reliability of the subjective estimation of cuff inflation up to a clinically acceptable degree, we performed an investigation similar to that by Michlig [5], using a modified tracheal tube in which the pilot balloon was replaced by a second cuff, identical to the one which would be inflated in the trachea.

\section{Methods}

Two size-9 Rüschelit ${ }^{\circledR}$ tracheal tubes (Teleflex Medical, Wayne, PA) were used to build the 'modified tracheal tube' shown in Fig. 1. Thanks to a Y-shaped tubing system equipped with a one-way valve, the (identical) cuffs of the two tubes were connected to each other, so as to inflate one of them into a simulated trachea while using the other one

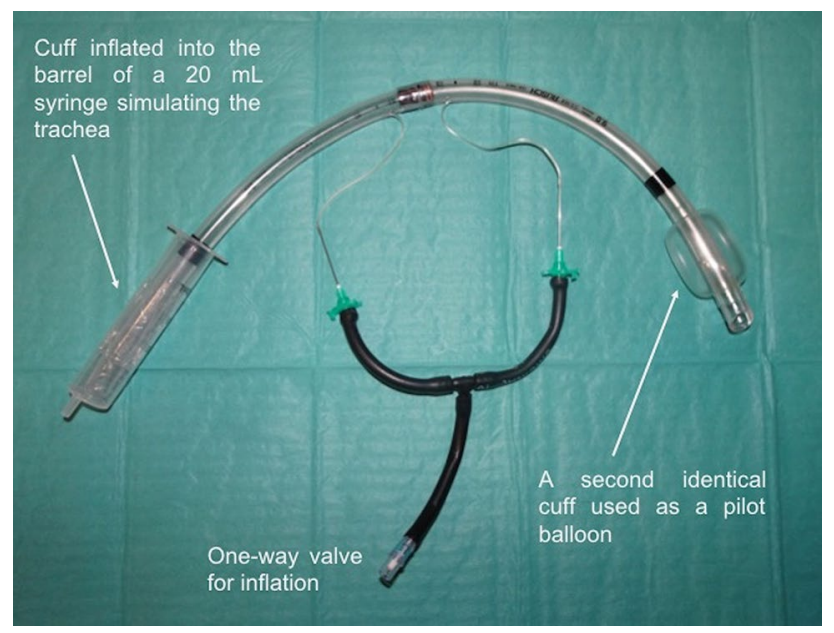

Fig. 1 The modified endotracheal tube with 'large' pilot balloon as a 'large' pilot balloon. One side of this modified tube was inserted into the barrel of a $20 \mathrm{~mL}$ syringe, and the cuff was inflated to $88 \mathrm{mmHg}$ using a cuff pressure gauge VBM (Bosco Medical Australia, Murarrie, Queensland) connected to the one-way valve located on the Y-piece. Anaesthesia practitioners (including both staff anaesthesiologists with at least 5 years of experience as specialists and anaesthesia residents) from two high-specialty hospitals and one University hospital were asked to palpate the second cuff as a pilot balloon and say if the pressure was 'too high', 'adequate', or 'too low'. Similarly, a normal size-9 Rüschelit tracheal tube was inserted into the barrel of a $20 \mathrm{~mL}$ syringe, its cuff was inflated to $88 \mathrm{mmHg}$, and participants were asked to estimate the cuff pressure by feeling the usual pilot balloon, again saying whether it was 'too high', 'adequate', or 'too low'. If the answer was the same with both the normal and the 'large' pilot balloon, participants were asked to state in which of the two systems they though the pressure was higher. Subsequently, the pressure in the 'modified tube' cuff was reduced to $40 \mathrm{mmHg}$, and participants were again asked to feel the 'large' pilot balloon and indicate whether the pressure was 'too high', 'adequate', or 'too low'. All participants were unaware of the actual pressures within the cuffs, both before and after the interview.

As in the study by Michlig [5], answers were grouped in correct ('too high') and incorrect ('adequate' or 'to low') and analysed with the $\mathrm{X}^{2}$ test with Yates' correction (overall data) or the Fisher's exact test (subgroups of experienced anaesthesiologists and residents) in order to verify if the answers provided after palpation were statistically different as compared with random answers. The same statistical tests were used to compare the three possible answers provided after palpation of the normal pilot balloon with those provided after palpation of the 'large' pilot balloon, in order to test the hypothesis that the accuracy of the 'finger-pressure' method in recognizing a cuff overinflated to $88 \mathrm{mmHg}$ was significantly higher with the 'large' pilot balloon. Finally, the answers about which of the two systems (both inflated to $88 \mathrm{mmHg}$ ) had the higher pressure (including when both were felt as 'too high'), as well as the answers about the cuff inflated to $40 \mathrm{mmHg}$, were similarly analysed. Version 22.0 (2013) of the IBM SPSS Statistics software (IBM, Armonk, NY) was used for data analysis. $P$ values $<0.05$ were considered statistically significant.

\section{Results}

Overall, 62 colleagues (41 experienced staff anaesthesiologists and 21 anaesthesia residents) participated in the study, and their answers are reported in Table 1. Similarly to what reported by Michlig [5], only 35\% of participants (and 49\% of experienced anaesthesiologists) recognized considerable 
Table 1 Answers of the 62 participants (overall and by subgroup)

Normal pilot bal- 'Large' pilot bal- 'Large' pilot balloon $(88 \mathrm{mmHg}) \quad$ loon $(88 \mathrm{mmHg})$ loon $(40 \mathrm{mmHg})$

\begin{tabular}{lccc}
\hline \multicolumn{3}{l}{ Overall $(n=62)$} \\
Too much & $22(35 \%)$ & $54(87 \%)$ & $20(32 \%)$ \\
Adequate & $26(42 \%)$ & $6(10 \%)$ & $24(39 \%)$ \\
Too low & $14(23 \%)$ & $2(3 \%)$ & $18(29 \%)$ \\
Experienced staff anaesthesiologists $(n=41)$ & \\
Too much & $20(49 \%)$ & $39(95 \%)$ & $21(51 \%)$ \\
Adequate & $18(44 \%)$ & $2(5 \%)$ & $16(39 \%)$ \\
Too low & $3(7 \%)$ & $0(0 \%)$ & $4(10 \%)$ \\
Anaesthesia residents $(n=21)$ & & $0(0 \%)$ \\
Too much & $2(10 \%)$ & $15(71 \%)$ & $7(33 \%)$ \\
Adequate & $8(38 \%)$ & $4(19 \%)$ & $14(67 \%)$ \\
Too low & $11(52 \%)$ & $2(10 \%)$ & \\
\hline
\end{tabular}

overinflation ( $88 \mathrm{mmHg}$ ) by palpation of the usual pilot balloon. The $\mathrm{X}^{2}$ test $(P=0.85)$ and the Fisher's exact test $(P=0.19)$ confirmed that the answers did not significantly differ from random answers in the overall population and in the subgroup of experienced anaesthesiologists, respectively. Nineteen out of 21 residents (90\%) did not recognize overinflation, although Fisher's test did not reach statistically significance in relation to the hypothesis that answers were different from those that would have been given at random $(P=0.075)$.

When colleagues were asked to evaluate the cuff inflated to $88 \mathrm{mmHg}$ by feeling the 'large' pilot balloon, $87 \%$ of participants, $95 \%$ of experienced anaesthesiologists, and $71 \%$ of residents recognized overinflation, with answers being significantly different from random answers in all three cases $(P<0.0001, P<0.0001$, and $P=0.017$, respectively). The $\mathrm{X}^{2}$ test confirmed that the answers provided after palpation of the 'large' pilot balloon were significantly different as compared with those provided after palpation of the normal pilot balloon $(\mathrm{P}<0.00001)$. Also the Fisher's exact test applied to the subgroup of experienced anaesthesiologists and to that of residents revealed that the answers were significantly different with the two different pilot balloons $(P<0.0001$ and $P=0.0001$, respectively). Moreover, 55 out of 62 participants $(89 \%), 35$ out of 41 experienced staff anaesthesiologists (85\%), and 20 out of 21 residents $(95 \%)$ believed that pressure within the larger pilot balloon was higher than in the normal pilot balloon (Fig. 2). Only four experienced anaesthesiologists (6\% of participants or $10 \%$ of experienced anaesthesiologists) and none of the residents recognized that the pressure in the two systems was the same, while $5 \%$ of participants (overall and among subgroups) believed that the pressure was higher in the normal pilot balloon. Statistical analysis confirmed that these answers were significantly different from random answers $(P<0.0001)$.

When the cuff was inflated to $40 \mathrm{mmHg}$ and participants were asked to estimate its pressure by feeling the 'large' pilot balloon, only $32 \%$ of participants and $51 \%$ of experienced anaesthesiologists recognized slight overinflation, with answers not significantly different from random answers ( $P=0.85$ and $P=0.127$, respectively). None of the 21 residents recognized overinflation, and their answers would have been significantly more accurate if given at random $(\mathrm{P}=0.0043)$.

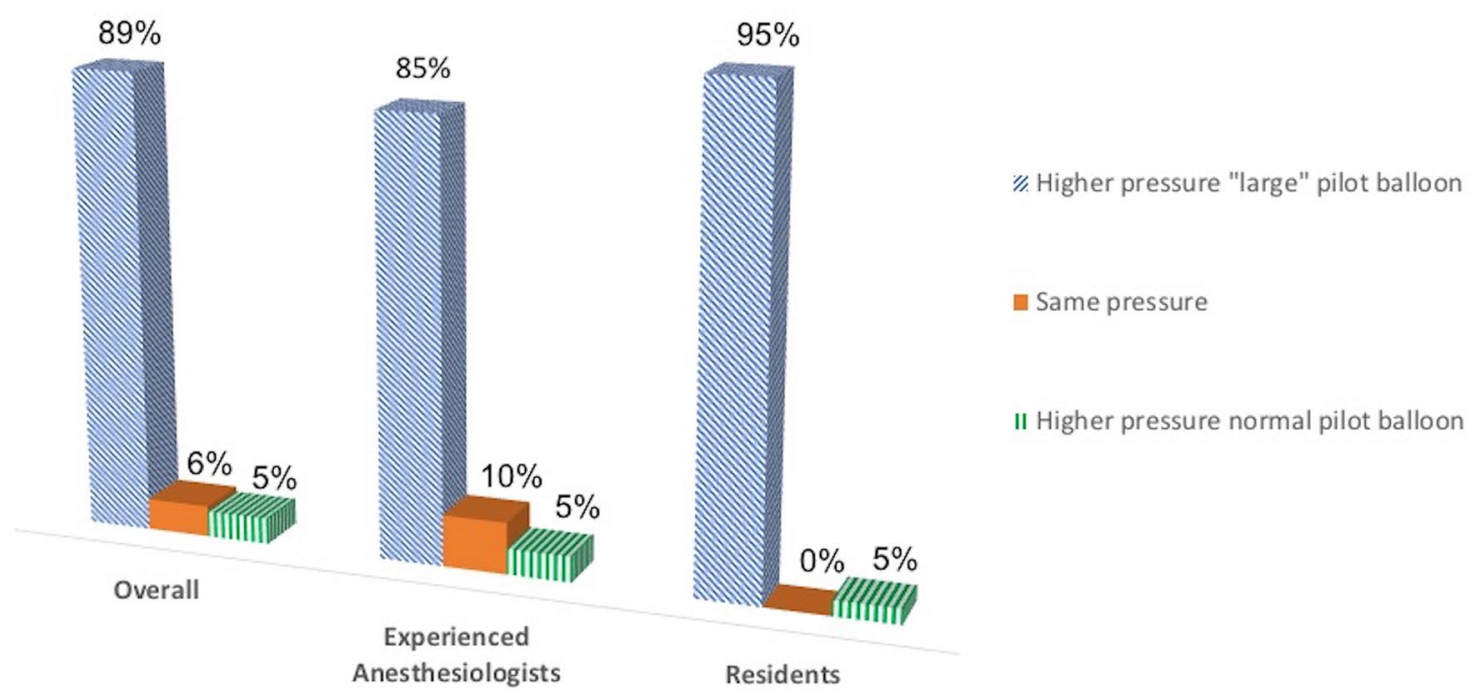

Fig. 2 Answers of the 62 participants (overall and by subgroup) to the question "in which system is the pressure higher?" (with both systems inflated to $88 \mathrm{mmHg}$ ) 


\section{Discussion}

While the results of the test with the normal tube suggest that our study population was similar to that investigated by Michlig [5], and confirm that pilot balloon palpation (with the conventional pilot balloon) is highly inaccurate in estimating the cuff pressure, even when performed by experienced clinicians, the results of the test with the 'modified tube' support the hypothesis that this poor accuracy is due, at least in part, to a 'structural' limitation, i.e. the different dimensions of the pilot balloon as compared with the cuff, as previously speculated [10,11]. It is particularly suggestive that, although the pressure in the two systems was the same, the large majority of participants 'felt' a higher pressure in the 'large' pilot balloon. This is consistent with the assumption that what clinicians really rely on to estimate the cuff pressure is the wall tension of the pilot balloon, which is higher for the 'large' pilot balloon according to Laplace's law. The residents' answers are not so surprising: due to their lack of experience, they are more easily misled by the lower wall tension of the pilot balloon, while they become much more able in detecting overinflation when the 'deception' is removed.

Although the accuracy of the 'finger-pressure' technique could be theoretically improved with the use of larger pilot balloons, our results suggest that, even completely eliminating the differences between the cuff and the pilot balloon, about one-half of experienced anaesthesiologists would still inflate the cuff to a pressure at least double than the recommended values, with possible serious harms. These findings are consistent with previous investigations which suggested that experience alone is not sufficient to ensure adequate cuff inflation [13] and emphasized the need for specific training [13] and, most importantly, for objective methods to monitor and optimize intracuff pressure [2, 3, 14]. Measurement of intracuff pressure by means of a pressure gauge to maintain values within a definite (safe) range is easy, rapid, and cheap. In addition to common aneroid pressure gauges, syringes equipped with coloured markers [15] or with a digital display [16] which allow acceptable estimation of intracuff pressure are available. Finally, a recent investigation suggested that the use of a closed-loop system which automatically adjusts cuff pressure according to the carbon dioxide levels in the subglottic space (as a marker of leakage) may be superior as compared to maintain intracuff pressure within a prefixed range using a common manual pressure gauge [2]. Despite the new insights on the possible 'physical' (and partly modifiable) reasons for the poor reliability of the 'finger-pressure' method, the present investigation confirms that objective measurement of cuff pressure, whatever the method used, should definitely replace subjective evaluation by pilot balloon palpation.
This study has several limitations, including the small sample size, the use of a very simplified model of trachea, and not taking into consideration the different material of which the normal pilot balloon and the cuff are made, as well as the different thickness of their walls, which may both affect the accuracy of the 'finger-pressure' estimation of cuff pressure [8]. For example, also a reduced wall thickness increases wall tension for a given pressure, according to Laplace's law [11]. Moreover, it could be argued that even the most experienced anaesthesiologists have not a touchstone when they feel the pilot balloon if they have never used a cuff pressure gauge. Accordingly, an adequately designed investigation of the impact of a larger pilot balloon in association with a specific training (involving the use of a pressure gauge for a number of times) on the accuracy of the 'fingerpressure' method could be interesting, in theory. However, as mentioned, objective measurement of intracuff pressure is easy, rapid, and cheap, and it is probably not worth trying to improve a method (to be considered, by now, obsolete) which will always remain, by definition, less accurate.

In conclusion, this study supports the difference between the cuff and the pilot balloon sizes as a major determinant of the poor ability of clinicians in recognizing cuff overinflation by pilot balloon palpation, due to the different wall tension according to Laplace's law. At the same time, it shows that removing such difference does not increase the reliability of the 'finger-pressure' method up to a clinically acceptable degree. Accordingly, objective measurement of intracuff pressure should definitely replace subjective pilot balloon assessment in all operating rooms.

Funding This work was supported only by institutional funds of the Department of Critical Care, AORN 'Dei Colli', Naples, Italy.

\section{Compliance with ethical standards}

Conflict of interest The authors declare that they have no conflict of interest.

\section{References}

1. Liu J, Zhang X, Gong W, Li S, Wang F, Fu S, Zhang M, Hang Y. Correlations between controlled endotracheal tube cuff pressure and postprocedural complications: a multicenter study. Anesth Analg. 2010;111(5):1133-7.

2. Efrati S, Bolotin G, Levi L, Zaaroor M, Guralnik L, Weksler N, et al Levinger U, Soroksky A, Denman WT, Gurman GM. Optimization of endotracheal tube cuff pressure by monitoring $\mathrm{CO}_{2}$ levels in the subglottic space in mechanically ventilated patients: a randomized controlled trial. Anesth Analg. 2017;125(4):1309-15.

3. Hockey CA, van Zundert AA, Paratz JD. Does objective measurement of tracheal tube cuff pressures minimise adverse effects and maintain accurate cuff pressures? A systematic review and meta-analysis. Anaesth Intensive Care. 2016;44(5):560-70. 
4. Giusti GD, Rogari C, Gili A, Nisi F. Cuff pressure monitoring by manual palpation in intubated patients: how accurate is it? A manikin simulation study. Aust Crit Care. 2017;30(4):234-8.

5. Michlig SA. Anaesthetic staff cannot identify extremely high tracheal tube cuff pressures by palpation of the pilot balloon. Br J Anaesth. 2013;111(2):300-1.

6. Sole ML, Penoyer DA, Su X, Jimenez E, Kalita SJ, Poalillo E, Byers JF, Bennett M, Ludy JE. Assessment of endotracheal cuff pressure by continuous monitoring: a pilot study. Am J Crit Care. 2009; 18:133-43.

7. Svenson JE, Lindsay MB, O'Connor JE. Endotracheal intracuff pressures in the ED and prehospital setting: is there a problem? Am J Emerg Med. 2007;25:53-6.

8. Janossy KM, Pullen J, Young D, Bell G. The effect of pilot balloon design on estimation of safe tracheal tube cuff pressure. Anaesthesia. 2010;65(8):785-91.

9. Hoffman RJ, Rarwani V, Hahn IH. Experienced emergency medicine physicians cannot safely inflate or estimate endotracheal tube cuff pressure using standard techniques. Am J Emerg Med. 2006;24:139-42.

10. Pisano A. Pitfalls from physics: why we can't "feel" the tube cuff pressure with our fingers. Anesth Analg. 2017;124(4):1368.
11. Pisano A. Bubbles, tracheal tube cuffs, and reservoir bags: surface tension and Laplace's law. In: Physics for anesthesiologists. Cham: Springer; 2017. pp. 33-43.

12. Pisano A. Toothpaste, sea deeps, and invasive pressure monitoring: Stevin's law and Pascal's principle. In: Physics for anesthesiologists. Cham: Springer; 2017. pp. 65-73.

13. Özcan ATD, Döğer C, But A, Kutlu I, Aksoy ŞM. Comparison of endotracheal tube cuff pressure values before and after training seminar. J Clin Monit Comput. 2018;32(3):527-31.

14. Efrati S, Deutsch I, Gurman GM. Endotracheal tube cuffsmall important part of a big issue. J Clin Monit Comput. 2012;26(1):53-60.

15. Kamata M, Kako H, Ramesh AS, Krishna SG, Tobias JD. An in vitro and in vivo validation of a novel color-coded syringe device for measuring the intracuff pressure in cuffed endotracheal tubes. Int J Clin Exp Med. 2015;8(7):11356-9.

16. Ramesh AS, Krishna SG, Denman WT, Tobias JD. An in vitro and in vivo validation of a novel monitor for intracuff pressure in cuffed endotracheal tubes. Paediatr Anaesth. 2014;24:1005-8. 